ИЗВЕСТИЯ АКАДЕМИИ НАУК ЭСТОНСКОИ ССР. ФИЗИКА * МАТЕМАТИКА

PROCEEDINGS OF THE ACADEMY OF SCIENCES OF THE ESTONIAN SSR. PHYSICS * MATHEMATICS

$1989,38,2$

УДК $517.948: 513.88$

O. VAARMANN

\title{
SOLUTION OF NONLINEAR LEAST SQUARES PROBLEMS BY LEVENBERG-MARQUARDT TYPE METHODS
}

\author{
(Presented by G. Vainikko)
}

This paper is concerned with minimization of a functional

$$
\varphi(x)=\|F(x)\|^{2},
$$

where $F$ is a Frechet differentiable operator which maps one Hilbert space, $H_{1}$, into another, $H_{2}$, and its derivative has the bounded pseudoinverse $\left[F^{\prime}(x)\right]^{+}$. A necessary condition for $x^{*}$ to be a local minimum point of $\varphi$ is

$$
\left[F^{\prime}(x)\right]^{*} F(x)=0 .
$$

To solve the equation (2) we consider methods in the form

$$
x_{k+1}=x_{k}-\varepsilon_{h} A_{k} F\left(x_{k}\right), \quad k=0,1, \ldots,
$$

where $\boldsymbol{\varepsilon}_{k}$ is a relaxation (damping) parameter $\left(0<\boldsymbol{\varepsilon}_{k} \leqslant 1\right), A_{k}$ is a linear operator from $H_{2}$ into $H_{1}$ approximating the operator $U^{-1}\left(F^{\prime}(x) U^{-1}\right)^{+}$, and $U$ is an arbitrary nonsingular linear operator from $H_{1}$ into itself. In the case $\varepsilon_{k}=1$ and $A_{k}=U^{-1}\left(F^{\prime}\left(x_{k}\right) U^{-1}\right)^{+}$one gets an extension of Ben-Israel method which coincides with the usual Ben-Israel method if $U_{i}=I$, where $I$ denotes identity mapping. In keeping with the terminology of [1] the operator $U^{-1}\left(F^{\prime}(x) U^{-1}\right)^{+}$can be called the $I U$-weighted pseudoinverse of $F^{\prime}(x)$ and, in general case $U$ is allowed $[1,2,3]$ to be a singular operator. Note that the introduction of the nonsingular operator $U$ into the computational scheme (3) is not principal but it is due to the convenience of the treatment of some problems in numerical practice $\left[{ }^{1,3}\right]$. The solution of a problem depends on the spaces considered. Via a change in the topology, the initial problem can be converted into one with $U=I$. The philosophy underlying many methods of regularization lies in deciding in what sense one should understand an approximate solution of an ill-posed problem.

The main aim of this paper is to study the convergence properties of various methods based on solving the normal equation within a single framework of the notion of the $I U$-weighted pseudoinverse. Introduction of the damping parameter $\varepsilon$ is a common strategy for enlarging the domain of convergence and sometimes also for converging acceleration in the sense of the required bulk of iterations or run time in seconds on the computer $\left[{ }^{4,5}\right]$.

The family of methods (3) involves in particular cases underrelaxed or damped Levenberg-Marquardt type methods as follows

$$
x_{k+1}=x_{k}-\varepsilon_{k} D_{k}\left[F^{\prime}\left(x_{k}\right)\right]^{*} F\left(x_{k}\right), \quad k=0,1, \ldots,
$$

where $D_{k}$ is an approximation to $M^{-1}\left(x_{k}, \alpha_{k}\right):=\left\{\left[F^{\prime}\left(x_{k}\right)\right]^{*} F^{\prime}\left(x_{k}\right)+\alpha_{k} H\right\}^{-1}$ with $\alpha_{k}>0$ and $A_{k}:=D_{k}\left[F^{\prime}\left(x_{k}\right)\right]^{*}$. It is assumed that $H$ is represented in the form $H=U^{*} U$. In order to guarantee the global convergence for 
Levenberg-Marquardt (regularized Gauss-Newton) type methods, the regularization parameter $\alpha$ used in [ $\left.{ }^{4}\right]$ is chosen from the considerations that operators $M(\cdot, \alpha)$ appear to be positive definite ones. Here for obtaining the convergence for methods (3) from a poor starting point $x_{0}$ operators $A_{k}$ need not necessarily be positive definite.

Great values of parameter $\alpha$ can lower the convergence. On the other hand, tending the parameter $\alpha$ to zero can cause a numerical instability. This is the reason why we are interested in the variants of LevenbergMarquardt type methods with successive approximation of the inverse (pseudoinverse) operator using only linear operators multiplications because the formula (17) appears to be self-correcting one with $\alpha>0$. Moreover, methods (3) based on formula (17) do not break down even with $\alpha=0$, provided operators $F^{\prime}(x)$ and $F^{\prime}(y)$ are in the acute case for all $x$ and $y$ belonging to a region under discussion $\left.{ }^{6}\right]$.

1. Since there exists the bounded pseudoinverse, then the operator $F^{\prime}(x)$ has the closed range $R(x)=R\left(F^{\prime}(x)\right)$.

Let

$$
\Phi(x)=\left[F^{\prime}(x) U^{-1}\right]^{+}, \quad \Psi(x)=\Phi^{+}(x)=F^{\prime}(x) U^{-1},
$$

and let $P_{R(x)}=P_{R(\Psi(x))}\left(P_{k}=P_{R\left(\Psi\left(x_{k}\right)\right)}\right)$ denote the orthogonal projector of $H_{2}$ onto $R(\Psi(x))$. Let further $C, \bar{C}, \bar{L}_{0}, L_{1}, N, N_{0}, N^{\prime}{ }_{1}, N_{2}$ and $L$ denote some positive constants. Supposing that $\bar{A}_{k}$ is an approximation to $\left[F^{\prime}\left(x_{k}\right) U^{-1}\right]^{+}$, i. e. $\bar{A}_{k}=U A_{k}$ and the following relations hold

$$
\bar{A}_{k}=\bar{A}_{k} P_{k}, \quad\left\|\bar{A}_{k}\right\| \leqslant \bar{\lambda}_{k} \leqslant \bar{\lambda}<\infty, \quad\left\|A_{k}\right\| \leqslant \lambda_{k} \leqslant \lambda<\infty ;
$$

using the results ( $\left.{ }^{7}\right]$ p. $195-197$ ), it is not difficult to prove the following assertion.

Le mma 1. Let $F^{\prime}(x)$ be Lipschitz continuos and $\Phi(x)$ be uniformly bounded on some set $S$, i.e.

$$
\begin{array}{ll}
\left\|F^{\prime}(x)-F^{\prime}(y)\right\| \leqslant L_{1}\|x-y\|, & \forall x, y \in S, \\
\|\Phi(x)\| \leqslant \bar{C}, & \forall x \in S,
\end{array}
$$

then

$$
\left\|P_{R(x)}-P_{R(y)}\right\| \leqslant \bar{L}_{0}\|x-y\|, \quad \forall x, y \in S,
$$

where $\bar{L}_{0}=\bar{C} L_{1}\left\|U^{-1}\right\|$.

Proof of lemma 1 is similar to that in [ $\left.{ }^{5}\right]$.

Remark 1 . From Lemma 1 it follows that there exist scalars $N^{\prime}$ and $N$ such that

$$
\begin{gathered}
\left\|\left(P_{R(y)}-P_{R(y)} P_{R(x)}\right) F(x)\right\|=\left\|\left(P_{R(y)}-P_{R(x)}\right)\left(I-P_{R(x)}\right) F(x)\right\| \leqslant \\
\leqslant N^{\prime}\|x-y\|, \quad x, y \in S,
\end{gathered}
$$

and

$$
\left\|\left(P_{k+1}-P_{k+1} P_{k}\right) F\left(x_{k}\right)\right\| \leqslant \varepsilon_{k} N\left\|P_{k} F\left(x_{k}\right)\right\|, \quad x_{k}, x_{k+1} \in S,
$$

where $\sup _{x \in S}\left\|\left(I-P_{R(x)}\right) F(x)\right\|<N^{\prime \prime}, N^{\prime}=N^{\prime \prime} \bar{L}_{0}$, and $N=N^{\prime} \lambda$. It can be readily shown that if $F$ satisfies Lipschitz condition with some constant $L_{1}$ then $\Psi$ satisfies Lipschitz condition with the constant $\bar{L}_{1}=L_{1}\left\|U^{-1}\right\|$.

2. Let $\bar{A}_{k}=U A_{k}$. Introducing a sequence $\left\{\bar{\gamma}_{k}\right\}$ defined as

one gets

$$
\left\|P_{k}-\Psi\left(x_{k}\right) \bar{A}_{k}\right\| \leqslant \bar{\gamma}_{k}, \quad k=0,1, \ldots,
$$


Theorem 1. Let $x_{0} \in H_{1}, S=\left\{x \in H_{1}:\left\|x-x_{0}\right\| \leqslant \varrho\right\}$, and the following conditions be valid in S:

$1^{\circ}$ operator $F$ has Frechet-derivative $F^{\prime}$ :

$2^{\circ}$ derivative $F^{\prime}$ satisfies Lipschitz condition $\left\|F^{\prime}(x)-F^{\prime}(y)\right\| \leqslant L_{1}\|x-y\|$

$3^{\circ} \delta=\delta_{0}<1$ (below $\delta_{0}$ is defined differently in the cases $A$ and $\left.B\right)$;

$4^{\circ} 0<\varepsilon_{0} \leqslant \varepsilon_{k-1} \leqslant \varepsilon_{k}=\min \left\{1, \varepsilon_{k-1} \delta^{-1 / 2}\right\}$.

A. If there exists a constant $\bar{C}$ such that $\|\Phi(x)\| \leqslant \bar{C}, \bar{\gamma}_{k} \leqslant \bar{\gamma}_{0}$ and $r_{1}=$ $=\lambda\left\|P_{0} F\left(x_{0}\right)\right\| /(1-\delta) \leqslant 0$, then the sequence $\left\{x_{k}\right\}$ generated by the method (3) has a limit $x^{*}$ which appears to be a solution of the equation $\left[F^{\prime}(x)\right]^{*} F(x)=0$ with $\left\|x^{*}-x_{0}\right\| \leqslant r_{1}, \delta_{k} \leqslant \delta$, and $\left\|x_{k}-x^{*}\right\| \leqslant r_{1} \delta^{k}$, where $\delta=\delta_{0}=1-\varepsilon_{0}+\varepsilon_{0}\left(\bar{\gamma}_{a}+N\right)+\frac{1}{2} \varepsilon_{0}^{2} \lambda^{2} L_{1}\left\|P_{0} F\left(x_{0}\right)\right\|$.

B. If operator $P_{R(x)}$ is independent of $x$ and $\bar{\gamma} \geqslant \bar{\gamma}_{0} \geqslant \ldots \geqslant \bar{\gamma}_{k} \geqslant \ldots \geqslant 0$ $\left(\bar{\gamma}_{k} \rightarrow 0\right.$ as $\left.k \rightarrow \infty\right)$, then the sequence $\left\{x_{k}\right\}$ converges superlinearly with $\left\|x^{*}-x_{0}\right\| \leqslant r_{1}$, and

$$
\left\|x_{k}-x^{*}\right\| \leqslant r_{1} \prod_{i=0}^{k-1} \delta_{i}
$$

where $\delta_{i}=1-\varepsilon_{i}+\varepsilon_{i} \bar{\gamma}_{i}+\frac{1}{2} \varepsilon_{i}^{2} \lambda^{2} L_{1}\left\|P_{i} F\left(x_{i}\right)\right\| \rightarrow 0$ as $i \rightarrow \infty$.

Proof. Denote $\varphi_{k}(\varepsilon)=\varepsilon \psi_{k}+1-\varepsilon$, where $\psi_{k}$ are some real numbers. If $\psi_{k} \leqslant \psi<1$ for all $k=0,1, \ldots$, then $\frac{d}{d \varepsilon} \varphi_{k}(\varepsilon)=\psi_{k}-1 \leqslant \psi-1<0$ and hence $\varphi_{k}(\varepsilon)$ is a decreasing function in $\varepsilon$.

Since $\left\|P_{k}-\varepsilon \Psi\left(x_{k}\right) \bar{A}_{k}\right\| \leqslant \varepsilon\left\|P_{k}-\Psi\left(x_{k}\right) \bar{A}_{k}\right\|_{1}+1-\varepsilon \leqslant \varepsilon \bar{\gamma}+1-\varepsilon$, in the capacity of $\bar{\gamma}_{k}(\varepsilon)$, one can take $\bar{\gamma}_{k}(\varepsilon) \triangleq \varepsilon \bar{\gamma}_{k}+1-\varepsilon$.

According to Taylor expansion one obtains

$$
\begin{gathered}
P_{k+1} F\left(x_{k+1}\right)=\left(P_{k+1}-P_{k+1} P_{k}\right) F\left(x_{k}\right)+P_{k+1}\left\{\left(P_{k}-\varepsilon_{k} \Psi\left(x_{k}\right) \bar{A}_{k}\right) F\left(x_{k}\right)+\right. \\
\left.+\varepsilon_{k} \int_{0}^{1}\left[F^{\prime}\left(x_{k}\right)-F^{\prime}\left(x_{k}+t\left(x_{k+1}-x_{k}\right)\right)\right] A_{k} F\left(x_{k}\right) \mathrm{dt} .\right\} .
\end{gathered}
$$

A. On the basis of the remark 1 one gets $\left\|P_{k+1} F\left(x_{k+1}\right)\right\| \leqslant \delta_{k}\left\|P_{k} F\left(x_{k}\right)\right\|$ where $\delta_{k}=\varepsilon_{k} N+\bar{\gamma}_{k}(\varepsilon)+\frac{1}{2} \varepsilon_{k}^{2} \lambda^{2} L_{1}\left\|P_{k} F\left(x_{k}\right)\right\|$. Supposing $\delta_{k} \leqslant \delta_{0}$, it will be shown that the relation $\delta_{k+1} \leqslant \delta_{0}$ holds as well.

Indeed in virtue of the preposition $4^{\circ} \varepsilon_{k+1}^{2} \delta_{k} \leqslant \varepsilon_{k+1}^{2} \delta \leqslant \varepsilon_{k}^{2}$ and $\left\|P_{k+1} F\left(x_{k+1}\right)\right\| \leqslant \delta_{k}\left\|P_{k} F\left(x_{k}\right)\right\|$ the following relations $\varepsilon_{k+1}^{2} \lambda^{2} L_{1} \times$ $X\left\|P_{k+1} F\left(x_{k+1}\right)\right\| \leqslant \varepsilon_{k+1}^{2} \lambda^{2} L_{1} \delta_{k}\left\|P_{k} F\left(x_{k}\right)\right\| \leqslant \varepsilon_{k}^{2} \lambda^{2} L_{1}\left\|P_{k} F\left(x_{k}\right)\right\|$ are valid. Setting now $\psi_{k}=\bar{\gamma}_{k}+N$ from the proposition $3^{\circ}$ and $\bar{\gamma}_{k} \leqslant \bar{\gamma}_{0}$, it follows that $\psi_{k} \leqslant \psi_{0}<1$.

Therefore $\varphi_{k+1}\left(\varepsilon_{k+1}\right) \leqslant \varphi_{k+1}\left(\varepsilon_{0}\right) \leqslant \varphi_{0}\left(\varepsilon_{0}\right)$ and

$$
\begin{gathered}
\delta_{k+1}=\varphi_{k+1}\left(\varepsilon_{k+1}\right)+\frac{1}{2} \varepsilon_{k+1}^{2} \lambda^{2} L_{1}\left\|P_{k+1} F\left(x_{k+1}\right)\right\| \leqslant \ldots \\
\ldots \leqslant \varphi_{0}\left(\varepsilon_{0}\right)+\frac{1}{2} \varepsilon_{0}^{2} \lambda^{2} L_{1}\left\|P_{0} F\left(x_{0}\right)\right\|=\delta_{0} .
\end{gathered}
$$

Further completion of the proof is similar to that in section B.

B. If $P_{R(x)}$ is independent of $x$, then $\delta_{k}=\bar{\gamma}_{k}\left(\varepsilon_{k}\right)+\frac{1}{2} \varepsilon_{k}^{2} \lambda^{2} L_{1}\left\|P_{k} F\left(x_{k}\right)\right\|$ and

$$
\bar{\gamma}_{k+1}\left(\varepsilon_{k+1}\right)=\varphi_{k+1}\left(\varepsilon_{k+1}\right) \leqslant \varphi_{k+1}\left(\varepsilon_{k}\right) \leqslant \varphi_{k}\left(\varepsilon_{k}\right)=\bar{\gamma}_{k}\left(\varepsilon_{k}\right) .
$$


If $\varepsilon_{k+1}^{2} \delta_{k} \leqslant \varepsilon_{k+1}^{2} \delta \leqslant \varepsilon_{k}^{2}$ or $\varepsilon_{k+1}=\varepsilon_{k}$, then one concludes by the induction that $\delta_{k+1} \leqslant \delta_{k} \leqslant \ldots \leqslant \delta_{0}<1$. The latter relations with the assumption $\bar{\gamma}_{k} \rightarrow 0$, while $k \rightarrow \infty$ implies that $\delta_{k} \rightarrow 0$. It is not difficult to see that (cf. $\left[{ }^{8}\right]$ )

$$
\begin{gathered}
\left\|x_{k+1}-x_{k}\right\| \leqslant \lambda_{k}\left\|P_{k} F\left(x_{k}\right)\right\| \leqslant \lambda_{k}\left\|P_{0} F\left(x_{0}\right)\right\| \prod_{i=0}^{k-1} \delta_{i} \leqslant \lambda\left\|P_{0} F\left(x_{0}\right)\right\| \delta^{k}, \\
\left\|x_{m}-x_{k}\right\| \leqslant r_{1}\left(\delta^{k}-\delta^{m}\right), \quad(m \geqslant k), \\
\left\|x_{k}-x_{0}\right\| \leqslant r_{1} \leqslant 0,
\end{gathered}
$$

Observing that

$$
\begin{aligned}
\left\|\left[F^{\prime}(x)\right]^{*} F(x)\right\| & \leqslant\|H\|\left\|H^{-1}\left[F^{\prime}(x)\right]^{*} F(x)\right\| \leqslant\|H\|\left\|U^{-1}\left(F^{\prime}(x) U^{-1}\right)^{*} F(x)\right\| \leqslant \\
& \leqslant\|H\|\left\|U^{-1}\left(F^{\prime}(x) U^{-1}\right)^{*} \Psi(x) \Phi(x) F(x)\right\| \leqslant \\
& \leqslant\|H\|\left\|U^{-1}\left(F^{\prime}(x) U^{-1}\right)^{*}\right\|\left\|P_{R(x)} F(x)\right\|
\end{aligned}
$$

and under the assumption of Theorem 1 the operator $P_{R(x)} F(x)$ is continuous, it is evident that $\left[F^{\prime}\left(x^{*}\right)\right]^{*} F\left(x^{*}\right)=0$.

$\mathrm{Remark} 2$. The rate of change for the parameter $\varepsilon$ is controlled by the condition $4^{\circ}$ of Theorem 1 which guarantees the validity of $\delta_{k} \leqslant \delta_{0}$ or $\delta_{k} \leqslant \delta_{k-1}$. It is obvious that the condition $4^{\circ}$ can be replaced by a less severe restriction $0<\varepsilon_{0} \leqslant \varepsilon_{k-1} \leqslant \varepsilon_{k}=\min \left\{1, \varepsilon_{k-1} \delta_{k-1}^{-1 / 2}\right\}$.

The condition $\delta=\delta_{0}<1$ can be rewritten as

$$
\varepsilon_{0}\left(\psi_{0}+\frac{1}{2} \varepsilon_{0} \lambda^{2} L_{1}\left\|P_{0} F\left(x_{0}\right)\right\|\right) \leqslant \varepsilon_{0},
$$

where $\psi_{0}=\bar{\gamma}_{0}+N$ and $\psi_{0}=\bar{\gamma}_{0}$ in the cases $A$ and $B$, respectively. Thus if $\psi_{0}<1$, then, clearly, by decreasing $\varepsilon_{0}$ one can accomplish that $\psi_{0}+\frac{1}{2} \varepsilon_{0} \lambda^{2} L_{1}\left\|P_{0} F\left(x_{0}\right)\right\|<1$.

It means that the condition $\delta_{0}<1$ can be guaranteed for all $x \in S$.

3. Now consider the case where in extension of Ben-Israel method instead of the derivative $F^{\prime}(x)$ a finite-difference approximation $\Delta F(x, h)$ $\left(h \in H_{1}\right)$ is used, i. e. $A_{k}=U^{-1}\left[\Delta F\left(x_{k}, h_{k}\right) U^{-1}\right]^{+}$. Let $C_{1}, \bar{C}_{1}, C_{2}$ and $C_{3}$ be some constants. Assuming $R\left(F^{\prime}(x)\right)=R(\Delta F(x, h)), \quad R\left(\left[F^{\prime}(x)\right]^{*}\right)=$ $=R\left([\Delta F(x, h)]^{*}\right) \quad\left(\left[{ }^{9}\right]\right.$ Section II. $\left.25,\left[{ }^{10}\right]\right)$ and $\left\|\bar{A}_{k}\right\|=\|\left[\Delta F\left(x_{k}, h_{k}\right) \times\right.$ $\left.\times U^{-1}\right]^{+} \leqslant \bar{C}_{1}$ for all $x \in S$, then

$$
\begin{gathered}
{[\Delta F(x, h)]^{+}=[\Delta F(x, h)]^{+} P_{R\left(F^{\prime}(x)\right)}=[\Delta F(x, h)]^{+} \Delta F(x, h)[\Delta F(x, h)]^{+},} \\
\left\|P_{k}-\psi\left(x_{k}\right) \bar{A}_{k}\right\|=\left\|\left(\Delta F\left(x_{k}, h_{k}\right)-F^{\prime}\left(x_{k}\right)\right) U^{-1} \bar{A}_{k}\right\|, \\
\left\|A_{k}\right\|=\left\|U^{-1} \bar{A}_{k}\right\| \leqslant\left\|U^{-1}\right\| \bar{C}_{1}=\lambda .
\end{gathered}
$$

If, in addition, the $\Delta F(x, h)$ is a «consistent approximation» to $F^{\prime}(x)$ ( $\left[{ }^{9}\right]$ p. $\left.344-352\right)$, then, from the assumption $2^{\circ}$ of Theorem 1 , it follows that

$$
\|\Delta F(x, h)-\Delta F(y, h)\| \leqslant L_{\Delta}\|x-y\|,
$$

where $L_{\Delta}$ is a constant (scalar).

Remark 3. If in the capacity of $\Delta F\left(x_{k}, h_{k}\right)$ to put a first order divided difference $F\left(y_{k} ; x_{k}\right)$ where the basic elements $x_{k}$ and $y_{k}$ satisfy the condition $\left\|y_{k}-x_{k}\right\| \leqslant C_{2}\left\|P_{k} F\left(x_{k}\right)\right\|$ and symmetric second order divided difference $F\left(y_{k} ; x_{k} ; x_{k}\right)$ is bounded with a constant say $K^{\prime}$, then one can take $\bar{\gamma}_{k}=C_{3}\left\|P_{k} F\left(x_{k}\right)\right\|$. 
Indeed,

$\left\|F\left(y_{k} ; x_{k}\right)-F^{\prime}\left(x_{k}\right)\right\|=\left\|F\left(y_{k} ; x_{k}\right)-F\left(x_{k} ; x_{k}\right)\right\| \leqslant\left\|F\left(y_{k} ; x_{k} ; x_{k}\right)\right\|\left\|y_{k}-x_{k}\right\|$,

$\left\|P_{k}-\Psi\left(x_{k}\right) \bar{A}_{k}\right\| \leqslant C_{3}\left\|P_{k} F\left(x_{k}\right)\right\|$ with $K^{\prime}=L_{\Delta}$ and $C_{3}=K^{\prime} C_{2} \lambda$.

4. Let $B(x)=\left[F^{\prime}(x)\right]^{*} F^{\prime}(x), B_{k}=B\left(x_{k}\right), M(x, \alpha)=B(x)+\alpha H, M_{k}=$ $=M\left(x_{k}, \alpha_{k}\right), L(x, \alpha)=M^{-1}(x, \alpha)\left[F^{\prime}(x)\right]^{*}$, where $H$ is a symmetric positive definite operator from $H_{1}$ into itself and $\alpha$ is a positive-valued scalar parameter.

For solving (2) one might use Levenberg-Marquardt-type methods

$$
x_{k+1}=x_{k}-\varepsilon_{k} D_{k}\left[F^{\prime}\left(x_{k}\right)\right]^{*} F\left(x_{k}\right),
$$

where $D_{k}$ is an approximation to $M_{k}^{-1}$ and $A_{k}=D_{k}\left[F^{\prime}\left(x_{k}\right)\right]^{*}$.

Let

$$
\omega_{k}=\mu_{k} / \alpha_{k}, \quad V=\|U\|\left\|H^{-1}\right\|
$$

and $\mu_{k}$ and $K$ be some scalars satisfying

$$
\left\|I-M_{k} D_{k}\right\| \leqslant \mu_{k}<1, \quad\left\|F^{\prime}(x)\right\| \leqslant K .
$$

Theorem 2. Let $x_{0} \in H_{1}, S=\left\{x \in H_{1}:\left\|x-x_{0}\right\| \leqslant \mathrm{Q}\right\}$, and let conditions $1^{\circ}-4^{\circ}$ be fulfilled in $S$.

A. If $\|\Phi(x)\| \leqslant \bar{C}, \quad \alpha_{k} \leqslant \alpha_{0}, \quad \omega_{k} \leqslant \omega_{0}\left(\alpha_{0}, \omega_{0}<\infty\right)$ and $r_{1}=\lambda\left\|P_{0} F\left(x_{0}\right)\right\| /$ $/(1-\delta) \leqslant \mathrm{Q}$, where $\delta=\delta_{0}=1-\varepsilon_{0}+\varepsilon_{0}\left[N+K\left\|U^{-1}\right\|\left(\alpha_{0} \bar{C}^{3}+\omega_{0} K\left\|H^{-1}\right\|\right)\right]+$ $+\frac{1}{2} \varepsilon_{0}^{2} \lambda^{2} L_{1}\left\|P_{0} F\left(x_{0}\right)\right\|$, then the sequence $\left\{x_{k}\right\}$ generated by the method

(14) has a limit $x^{*}$ which appears to be a solution of the equation (2) with $\left\|x^{*}-x_{0}\right\| \leqslant r_{1}$ and

$$
\left\|x_{k}-x^{*}\right\| \leqslant r_{1} \delta^{k} .
$$

B. If $P_{R(x)}$ is independent of $x, n \xi^{k} \leqslant \alpha_{k} \leqslant m \xi^{k}(0<m, n<\infty), 0<\xi<1$, $\omega_{k} \leqslant s l^{k}, s>0,0<l<1$, then the sequence (14) converges superlinearly with $\left\|x^{*}-x_{0}\right\| \leqslant r_{1}$ and

$$
\left\|x_{k}-x^{*}\right\| \leqslant r_{1} \prod_{i=0}^{k-1} \delta_{i}
$$

where $\quad \delta_{i}=1-\varepsilon_{i}+\varepsilon_{i} K\left\|U^{-1}\right\|\left(m \bar{C}^{3 \xi} \xi^{i}+s l i K\|U\|\left\|H^{-1}\right\|\right)+\frac{1}{2} \varepsilon_{i}^{2} \lambda_{i}^{2} L_{1} X$ $X\left\|P_{i} F\left(x_{j}\right)\right\| \rightarrow 0$ as $i \rightarrow \infty$.

Proof. Since

$$
\begin{aligned}
& \left\|\left(B_{k}+\alpha_{k} H\right)^{-1}\right\|=\left\|\left(H^{-1} B_{k}+\alpha_{k} I\right)^{-1} H^{-1}\right\| \leqslant \alpha_{k}^{-1}\left\|H^{-1}\right\|, \\
& \left\|L\left(x_{k}, \alpha_{k}\right)\right\|=\left\|M_{k}^{-1}\left[F^{\prime}\left(x_{k}\right)\right]^{*}\right\|=\left\|\left(H^{-1} B_{k}+\alpha_{k} I\right)^{-1}\left[F^{\prime}\left(x_{k}\right)\right]^{*}\right\|= \\
= & \left\|U^{-1}\left(U^{*-1} B_{k} U^{-1}+\alpha_{k} I\right)^{-1} U^{*-1}\left[F^{\prime}\left(x_{k}\right)\right]^{*}\right\| \leqslant\left\|U^{-1}\right\|\left\|\left[F^{\prime}\left(x_{k}\right) U^{-1}\right]+\right\|,
\end{aligned}
$$
then

$\left\|\Phi\left(x_{k}\right)-U L\left(x_{k}, \alpha_{k}\right)\right\|=\left\|\Phi\left(x_{k}\right)-\left(\Psi^{*}\left(x_{k}\right) \Psi\left(x_{k}\right)+\alpha_{k} I\right)^{-1} \Psi^{*}\left(x_{k}\right)\right\|=$ $=\left\|\left(\Psi^{*}\left(x_{k}\right) \Psi\left(x_{k}\right)+\alpha_{k} I\right)^{-1} \alpha_{k} \Phi\left(x_{k}\right)\right\|=$

$=\left\|\alpha_{k}\left(\Psi^{*}\left(x_{k}\right) \Psi\left(x_{k}\right)+\alpha_{k} I\right) \Psi^{*}\left(x_{k}\right) \Psi^{*+}\left(x_{k}\right) \Phi\left(x_{k}\right)\right\| \leqslant$ $\leqslant \alpha_{k}\left\|U L\left(x_{k}, \alpha_{k}\right)\right\|\|\bar{C}\|^{2}<\mid \alpha_{k} \bar{C}^{3}$,

$\left\|L\left(x_{k}, \alpha_{k}\right)-A_{k}\right\|=\left\|\left(B_{k}+\alpha_{k} H\right)^{-1}\left(I-M_{k} D_{k}\right)\left[F^{\prime}\left(x_{k}\right)\right]^{*}\right\| \leqslant \omega_{k} K\left\|H^{-1}\right\|$, $\left\|\bar{A}_{k}\right\|=\left\|\Phi\left(x_{k}\right)+U\left(L\left(x_{k}, \alpha_{k}\right)-L\left(x_{k}, \alpha_{k}\right)\right)+\bar{A}_{k}-\Phi\left(x_{k}\right)\right\| \leqslant$ $\leqslant \bar{C}+\left\|U\left(L\left(x_{k}, \alpha_{k}\right)-A_{k}\right)\right\|+\left\|U L\left(x_{k}, \alpha_{k}\right)-\Phi\left(x_{k}\right)\right\| \leqslant \bar{C}+\omega_{k} K V+\alpha_{k} \bar{C}^{3}$,

$\left\|P_{k}-\Psi\left(x_{k}\right) \bar{A}_{k}\right\|=\left\|\Psi\left(x_{k}\right)\left[\Phi\left(x_{k}\right)-\bar{A}_{k}\right]\right\|=\| \Psi\left(x_{k}\right)\left[\Phi\left(x_{k}\right)-\right.$ $-U\left(L\left(x_{k}, \alpha_{k}\right)-L\left(x_{k}, \alpha_{k}\right)\right)-\bar{A}_{k}\|\leqslant K\| U^{-1} \|\left(\alpha_{k} \bar{C}^{3}+\omega_{k} K V\right)$. 
In the case of $\mathrm{A}$ and $\mathrm{B}$, one can take, respectively,

$$
\begin{aligned}
& \bar{\lambda}=\bar{C}+\alpha_{0} \bar{C}^{3}+\omega_{0} K V, \\
& \bar{\lambda}=\bar{C}+m \bar{C}^{3}+s K V,
\end{aligned}
$$

and

$$
\begin{aligned}
& \bar{\gamma}_{k}=\alpha_{k} \bar{C}^{3} K\left\|U^{-1}\right\|+\omega_{k} K^{2} V \mid U^{-1} \|, \\
& \bar{\gamma}_{k}=m \xi^{k} \bar{C}^{3} K\left\|U^{-1}\right\|+s l^{k} K^{2} V\left\|U^{-1}\right\| .
\end{aligned}
$$

Existence of scalar $K$ such that $\left\|F^{\prime}(x)\right\| \leqslant K$ follows from (7).

Since $\left\|I-M_{k} D_{k}\right\| \leqslant \mu_{k}<1$ and $\lim _{\alpha_{k} \rightarrow 0} M_{k}^{-1}\left[F^{\prime}\left(x_{k}\right)\right]^{*}=U^{-1}\left[F^{\prime}\left(x_{k}\right) U^{-1}\right]^{+}$, then $A_{k}=D_{k}\left[F^{\prime}\left(x_{k}\right)\right]^{*}$ approximates the $U^{-1} \Phi\left(x_{k}\right)$ and one can apply Theorem 1.

Remark 4. Regularizing properties of method (14) provided $D_{k}=$ $=M_{k}^{-1}$ and its connections with the regularization method by $\mathrm{A}$. N. Tikhonov are discussed in [ $\left.{ }^{11,12}\right]$.

Remark 5. Methods of type (14) can also be called methods «of two parametric damping» because $\varepsilon$ as well as $\alpha$ limit the change $x_{k+1}-x_{k}$. It is well known that under certain conditions LevenbergMarquardt method is globally convergent $\left[{ }^{4,13}\right]$. There exists sufficiently large $\alpha_{k}$ such that $\varepsilon_{k}$ can be chosen as $\varepsilon_{k}=1$ for all $k$. Computational difficulties arise with these methods when the operator $F(x)$ is badly nonlinear. Moreover, introducing the damping parameter $\varepsilon$ enables to sparse the amount of the arithmetic per iteration step, avoiding the need for solving repeatedly one linear equation or inverting one linear operator at an iteration step ( $\left[{ }^{4}\right]$ p. 258$)$.

5. Consider now variants Levenberg-Marquardt-type methods where $D_{k}$ is generated recurrently by the following prescription

$$
D_{k+1}=D_{k} \sum_{i=0}^{q-1}\left(I-M_{k+1} D_{k}\right)^{i}, \quad q \geqslant 2, \quad k=0,1, \ldots
$$

In addition to the quantities $\mu_{k}, \bar{\gamma}_{k}, \lambda_{k}, \bar{\lambda}_{k}$ and $\omega_{k}$ given by (5), (11), (15), (16) let us define $\sigma_{k}$ as follows

$$
\left\|I-M_{k+1} D_{k}\right\| \leqslant \sigma_{k}, \quad k=0,1, \ldots,
$$

and put

$$
\begin{aligned}
& \mu_{0}=\max \left\{\left\|I-M_{0} D_{0}\right\|, \eta^{q}\right\}, \quad \max \left\{\eta_{0}, \sigma_{0}\right\} \leqslant \eta, \quad \max \left\{\sigma_{i}, \xi\right\} \leqslant \zeta_{i}, \\
& \eta=\left(1+\sigma_{i}+\ldots+\sigma_{i}^{q-1}\right) \zeta_{i}, \quad \bar{\lambda}=\bar{\lambda}_{0}=\bar{C}+\alpha_{0} \bar{C}^{3}+\Omega K\|U\|\left\|H^{-1}\right\|, \\
& \Omega=\max \left\{\mu_{0} / \alpha_{0}, h^{q} / n \xi\right\}, \quad h=\max \left\{\eta^{q-1}+\Lambda, \mu_{0}+\Lambda\right\}, \\
& \Lambda=\left(2 \varepsilon_{0} K L_{1} \lambda\left\|P_{0} F\left(x_{0}\right)\right\|+(m-n \xi)\|H\|\right)\left\|D_{0}\right\| .
\end{aligned}
$$

Theorem 3. Let $x_{0} \in H_{1}, \quad S=\left\{x \in H_{1}:\left\|x-x_{0}\right\| \leqslant \mathrm{Q}\right\}$, and let, in addition to the conditions $1^{\circ}-4^{\circ}$ of Theorem 1 , the following conditions be fulfilled:

$$
\begin{array}{ll}
1^{\circ} \eta^{q} \leqslant \xi, \quad h^{q} \leqslant \eta & \text { and } \eta, h, \xi<1 ; \\
2^{\circ} n \xi^{k} \leqslant \alpha_{k} \leqslant m \xi^{k} & (0<m, n<\infty) .
\end{array}
$$

A. If the condition (7) holds, $r_{1}=\lambda_{0}\left\|P_{0} F\left(x_{0}\right)\right\| /(1-\delta) \leqslant \mathrm{Q}$, where $\delta=$ $=\delta_{0}=1-\varepsilon_{d}+\varepsilon_{0}\left[N+K\left\|U^{-1}\right\|\left(m \bar{C}^{3}+\Omega K\|U\|\left\|H^{-1}\right\|\right)\right]+\frac{1}{2} \varepsilon_{0}^{2} \lambda^{2} L_{1}\left\|P_{0} F\left(x_{0}\right)\right\|$, 
then the sequence $\left\{x_{k}\right\}$ generated by (14), (17) has a limit $x^{*}$ which appears to be solution of the equation (2) with $\left\|x^{*}-x_{0}\right\| \leqslant r_{1}$ and

$$
\left\|x_{k}-x^{*}\right\| \leqslant r_{1} \delta^{k} \text {. }
$$

B. If $P_{R(x)}$ is independent of $x$, then the sequence $\left\{x_{k}\right\}$ converges superlinearly with $\left\|x^{*}-x_{0}\right\| \leqslant r_{1}$ and

$$
\left\|x_{k}-x^{*}\right\| \leqslant r_{1} \prod_{i=0}^{k-1} \delta_{i}
$$

where

$$
\begin{gathered}
\delta_{i}=1-\varepsilon_{i}+\varepsilon_{i} K\left\|U^{-1}\right\|\left[m \bar{C}^{3} \xi^{i}+\Omega K\|U\|\left\|H^{-1}\right\|\left(\eta^{q} / \xi\right)^{i}\right]+ \\
+\frac{1}{2} \varepsilon_{i}^{2} \lambda_{i}^{2} L\left\|P_{i} F\left(x_{i}\right)\right\| \rightarrow 0 \text { as } i \rightarrow \infty .
\end{gathered}
$$

Proof. From (17), (18) it follows

$$
\left\|I-M_{k} D_{k}\right\| \leqslant\left\|I-M_{k} D_{k-1}\right\| q, \quad\left\|D_{k}\right\| \leqslant\left\|D_{0}\right\| \prod_{i=0}^{k-1} \frac{1-\sigma_{i}^{q}}{1-\sigma_{i}},
$$

and from the definition of $B_{k}$ and $M_{k}$ one concludes that

$$
\begin{aligned}
& \left\|\left(M_{k-1}-M_{k}\right) D_{k-1}\right\| \leqslant \|\left\{\left[F^{\prime}\left(x_{k}\right)\right]^{*}\left(F^{\prime}\left(x_{k-1}\right)-F^{\prime}\left(x_{k}\right)\right)+\right. \\
+ & \left.\left(\left[F^{\prime}\left(x_{k-1}\right)\right]^{*}-\left[F^{\prime}\left(x_{k}\right)\right]^{*}\right) F^{\prime}\left(x_{k-1}\right)+\left(\alpha_{k-1}-\alpha_{k}\right) H\right\} D_{k-1} \| \leqslant \\
\leqslant & \left(2 \varepsilon_{k-1} \lambda_{k-1} K L_{1}\left\|P_{k-1} F\left(x_{k-1}\right)\right\|+(m-n \xi)\|H\|\right)\left\|D_{0}\right\| \prod_{i=0}^{k-2} \frac{\left(1-\sigma_{i}^{q}\right)}{1-\sigma_{i}}, \\
& \left\|I-M_{k} D_{k-1}\right\| \leqslant\left\|I-M_{k-1} D_{k-1}\right\|+\left\|\left(M_{k-1}-M_{k}\right) D_{k-1}\right\| \leqslant \sigma_{k-2}^{q}+\Lambda \eta^{k-1} .
\end{aligned}
$$

According to (12), one gets

$$
\left\|P_{k+1} F\left(x_{k+1}\right)\right\| \leqslant \delta_{k}\left\|P_{k} F\left(x_{k}\right)\right\|,
$$

where

$$
\delta_{k}=1-\varepsilon_{k}+\varepsilon_{k}\left[N+K\left\|U^{-1}\right\|\left(m \bar{C}^{3} \xi^{k}+\Omega K V\right)\left(\eta^{q} / \xi\right)^{k}\right]+\frac{1}{2} \varepsilon_{k}^{2} \lambda_{k}^{2} L_{1}\left\|P_{k} F\left(x_{k}\right)\right\|
$$

and

$$
\delta_{k}=1-\varepsilon_{k}+\varepsilon_{k} K\left\|U^{-1}\right\|\left(m \bar{C}^{3 \xi^{k}}+\Omega K V\right)\left(\eta^{q} / \xi\right)^{k}+\frac{1}{2} \varepsilon_{k}^{2} \lambda_{k}^{2} L_{1}\left\|P_{k} F\left(x_{k}\right)\right\|
$$

in the cases of $A$ and $B$, respectively.

Setting

$$
\begin{aligned}
& \bar{\lambda}=\bar{C}+\alpha_{0} \bar{C}+\Omega K V, \\
& \bar{\gamma}_{k}=m \xi^{k} \bar{C}^{3} K\left\|U^{-1}\right\|+s l^{k} K^{2} V\left\|U^{-1}\right\|,
\end{aligned}
$$

and making use of $(21)-(24)$, one has

$$
\mu_{1}=\sigma_{0}^{q} \leqslant \mu_{0}, \quad \sigma_{0} \leqslant \mu+\Lambda \leqslant h, \quad \omega_{1}=\mu_{1} / \alpha_{1} \leqslant h^{q} / n \xi \leqslant \Omega .
$$

In the remainder the proof is similar to proofs corresponding to Theorems in $\left[{ }^{5,8}\right]$.

$\mathrm{R}$ e ma rk 6 . In a similar way as in ([14] Theorem 2) it can be shown that in the case $\varepsilon_{k}=1$ and $P_{R(x)}$ being independent of $x$ the sequence $\left\{x_{k}\right\}$ generated by the formulas (14) and (17) converges quadratically to a solution of (2). discussions. 
1. Elden, L. A note on weighted pseudoinverses with application to the regularization of Fredholm integral equations of the first kind. Report LitH - MAT $-\mathrm{R}-$ 1975 - 11, Department of Mathematics, Linköping University (Sweden), 1975.

2. Diaconu, A. // Mathematică (RSR), 1980, 22, № 2, 247-261.

3. Locker J., Prenter, P. M. // SIAM J. Numer. Anal., 1980, 17, 247-267.

4. Schwetlick, $H$. Numerische Lösung nichtlinear Gleichungen, Berlin, VEB Deutscher Verlag der Wissenschaften, 1979.

5. Ваарманн О., Ломп М. // Изв. АН ЭССР. Физ. Матем., 1982, 31, № 4, 410417.

6. Vaarmann, O. // Proc. Acad. Sci. ESSR. Phys. Math., 1978, 27, № 3, 251-258.

7. Красносельский М. А., Вайникко Г. М., Забрейко П. П., Рутицкий Я. Б., Стеценко B. Я. Приближенное решение операторных уравнений. М., Наука, 1969.

8. Ваарманн О. // Изв. АН ЭССР. Физ. Матем., 1970, 19, № 3, 265-274.

9. Ortega, J. M., Rheinboldt, W. C. Iterative Solution of Nonlinear Equations in Several Variables. New York; London, Academic Press, 1970.

10. Boggs, P. T., Dennis, J. E. jr. // Math. Comput., 1976, 30, 199-215.

11. Оганесян С. М., Старостенко В. И. // Изв. АН СССР. Физ. Земли, 1978, № 1, $63-75$.

12. Старостенко В. И., Оганесян С. М. // Методы решения некорректных задач и приложения (ред. А. Н. Тихонов). Нарва-Ыйэсуу; Москва, 1982, 119-128.

13. Marquardt, D. W. // SIAM J. Appl. Math., 1963, 11, 431-441.

14. Vaarmann, O. // Proc. Acad. Sci. ESSR. Phys. Math., 1980, 29, № 3, 233-240.

Academy of Sciences of the Estonian SSR, Institute of Cybernetics
Received

March 31, 1988

\section{O. VAARMANN}

\section{MITTELINEAARSETE VÄHIMRUUTUDE OLESANNETE LAHENDAMINE LEVENBERG-MARQUARDTI TOUPI MEETODITEGA}

Mittelineaarse operaatorvõrrandi üldistatud lahendi arvutamiseks on välja töötatud üks üldistatud pseudopöördoperaatori aproksimeerimisel baseeruv iteratsioonimeetodite pere, mis erijuhul sisaldab Gauss-Newtoni, Ben-Israeli, Levenberg-Marquardti meetodid, aga ka üldistatud pseudopöördoperaatori järkjärgulisel aproksimeerimisel põhinevad meetodid. Iteratsioonimeetodite koonduvusomaduste parandamiseks, sealhulgas koonduvuspiirkonna laiendamiseks, on kasutusele võetud iteratsioonisammu pikkust reguleerivad nn. relaksatsiooniparameetrid. On tōestatud koonduvusteoreemid selle iteratsioonimeetodite pere jaoks.

\section{O. BAAPMAHH}

\section{РЕШЕНИЕ НЕЛИНЕИНЫХ ЗАДАЧ НАИМЕНЬШИХ КВАДРАТОВ МЕТОДАМИ ТИПА ЛЕВЕНБЕРГА-МАРКВАРДТА}

Понятие псевдообратного оператора позволяет укладывать в единые рамки различные итерационные методы. В случае гильбертова пространства рассматриваются методы решения задач определения обобщенного решения нелинейного операторного уравнения в терминах обобщенной псевдоинверсии. Доказаны теоремы о сходимости для одного семейства итеращионных методов, включающих в себя как частные случаи методы Гаусса-Ньютона, Бен-Израэля, Левенберга-Марквардта, а также методы с последовательной аппроксимацией обобщенной псевдоинверсии. Установлена область допустимых значений релаксационного (шагового) параметра, позволяющего ослаблять ограничения на выбор начального приближения. 